\section{Noch eine unbequeme Wahrheit}

\author{
Ein Kreuzfahrtschiff verursacht während der Fahrt so viele \\ Schadstoffe wie 5.000.000 Autos. Während Autos, LKWs \\ und Diesellokomotiven zunehmend mit Abgastechnik \\ unterwegs sind, ist die Schifffahrt der letzte Verkehrsträger, \\ bei dem Ökonomie und Ökologie weiterhin im Widerspruch \\ stehen. \\ Von Dietmar Oeliger
}

$\mathrm{K}_{r}^{\mathrm{n}}$ reuzfahrtschiffe versprechen Traumziele, grenzenloses Reisevergnügen und einzigartige Urlaubserlebnisse. Auf ihnen sind so viele Menschen und so viele Restaurants untergebracht wie in einer Kleinstadt. Aber mit Schwimmbad, Casino oder Kino an Bord verbrauchen sie auch entsprechend viel Energie und stoßen Unmengen von Schadstoffen aus.

\section{Kein ausgeprägtes Problem- bewusstsein}

Die Nachfrage nach Kreuzfahrten steigt seit Jahren mit zweistelligen $\mathrm{Zu}$ wachsraten. Kaum ein Monat vergeht, in dem nicht irgendwo auf der Welt ein neuer Kahn vom Stapel läuft. Erst kürzlich ging der schwimmende Freizeitpark „Oasis of the Seas“, der größte Luxusliner aller Zeiten, von Florida aus auf Jungfernfahrt. Doch was kaum bekannt ist: Keiner dieser Ozeanriesen würde die Pkw-Abgasuntersuchung schaffen. Es ist paradox, das, was an Land schon lange kontrolliert und verboten ist, auf See weitgehend unreglementiert und ungestraft bleibt. Die Schifffahrtsindustrie nutzt so ein skandalöses Verschmutzungsprivileg.

Der sicherste Platz an Bord eines Kreuzfahrtschiffes ist deshalb nicht nur in der Nähe der Rettungsboote, sondern auch vor dem Abgasschornstein, denn dort atmet man weniger Abgase ein. Mittlerweile hat die Weltgesundheitsorganisation nachgewiesen, dass das, was dort aus den Schornsteinen kommt, krank macht und sogar krebserregend sein kann. Wenn die Schiffe in den Häfen vor Anker liegen, schalten sie nicht etwa die Motoren aus, so wie es für jeden Autofahrer auf einem Parkplatz normal ist, sondern lassen ihre Hilfsmotoren permanent und ungefiltert weiterlaufen.

Umgerechnet stößt ein Kreuzfahrtschiff so viele Schadstoffe aus wie 5.000.000 Autos während der Fahrt. Neben dem Ausstoß an Kohlendioxid führen insbesondere die Rußpartikel aus den Dieselmotoren $\mathrm{zu}$ einer zusätzlichen Klimaerwärmung, wie Wissenschaftler nachgewiesen haben. Für die Kreuzfahrt reedereien ist diese Erkenntnis eine unbequeme Wahrheit. Das Verantwortungsgefühl und der offene Umgang mit dem Abgasproblem sind bisher kaum ausgeprägt. Zu sehr steht das SaubermannImage der weißen Kreuzer auf dem Spiel. Wer eine Kreuzfahrt bucht, soll nicht an Umweltverschmutzung, an den Klimawandel oder gar an krebserregende Abgase erinnert werden.

Die Schiffe werden auf hoher See fast ausnahmslos mit Schweröl angetrieben, einem Abfallprodukt der Erdölverarbeitung. Die bisherigen Filter vertragen die Rückstände aus der Verbrennung dieses Substrats nicht. Deshalb gibt es Überlegungen, zumindest die Hilfsmotoren im Hafen mit Gas zu betreiben oder die Schiffe während der Liegezeiten an den Landstrom anzuschließen. Beides würde dazu führen, dass die Rußbelastung in den Häfen deutlich zurückginge. In Lübeck beispielsweise machen die Feinstaubemissionen der Schiffe 80 Prozent der Gesamtimmissionsbelastung aus. Auch die Ausweitung sogenannter „Emission Control Areas", also Umweltzonen auf See, wie es sie bereits an der Westküste der USA, vor Kanada sowie in der Nord- und Ostsee gibt, wird intensiv diskutiert.

\section{Reduzierung der Belastun- gen ist unkompliziert}

Die am schnellsten wirksame und technisch simpelste Maßnahme zur Rußreduzierung ist die Verwendung von schwefelarmen Treibstoffen auf hoher See sowie in Küsten- und Hafengebieten. Damit werden nicht nur giftige Schwefelund Stickoxid-Emissionen reduziert, sondern gleichzeitig auch der Rußausstoß. Diese Maßnahme könnten die Reeder sofort und ohne Ausrede umsetzen, nicht erst in einer fernen Zukunft. Die Kreuzfahrt-Reedereien setzen bisher jedoch ungeniert aus Profitgier weiter auf das billige Schweröl. So wird auch in Zukunft eine schwarze, gefährliche Wolke die weißen Riesen ständig begleiten. Dies würde uns über Umwege teuer zu stehen kommen, die Folgekosten durch Krankenbehandlung und Klimaveränderungen treffen die gesamte Gesellschaft.

Kreuzfahrtschiffe sind nur ein Teil des globalen Seeverkehrs, aber ein vergleichsweise sensibler. Kein Tourist möchte bewusst seinen Urlaub auf einer Dreckschleuder verbringen, wohingegen man sich deutlich weniger Gedanken darüber macht, mit welchem Frachtschiff ein Elektronikteil oder T-Shirt aus Asien importiert wurde. Umso wichtiger ist es, dass der politische und öffentliche Druck auf die Reedereien steigt. Denn was technisch und administrativ bei Kreuzfahrtschiffen gelingt, ist dann auch auf Containerschiffe übertragbar.

I AUTOR + KONTAKT

Dietmar Oeliger ist Leiter der Abteilung Verkehrspolitik beim Bundesverband des Naturschutzbund Deutschland (NABU).

E-Mail: Dietmar.Oeliger@NABU.de; Internet: www.nabu.de/kreuzfahrtschiffe; www.facebook.de/mirstinkts 


\section{Lizenzhinweis}

Die Beiträge in ÖkologischesWirtschaften werden unter der Creative-Commons-Lizenz "CC 4.0 Attribution Non-Commercial No Derivatives" veröffentlicht. Im Rahmen dieser Lizenz muss der Autor/Urheber stets genannt werden, das Werk darf nicht bearbeitet, abgewandelt oder in anderer Weise verändert und außerdem nicht kommerziell genutzt werden. Die digitale Version des Artikels bleibt für zwei Jahre Abonnent/innen vorbehalten und ist danach im Open Access verfügbar. 